\title{
An Efficient Proactive Routing Method for Mobile Ad Hoc Networks using Peer-to-Peer and Cellular Communication System
}

\author{
Hiroaki Morino $\nmid$, Tadao Saitołł and Mitsuo Noharał \\ $\nmid$ Research and Development Initiative Chuo University \\ $\ddagger$ TOYOTA InfoTechnology Center Co., Ltd \\ Address: 42-8 Hommuracho Shinjuku-ku Tokyo 162-8473, Japan \\ Tel: $+81-3-5368-3571$ \\ Fax: $+81-3-5368-3515$ \\ morino@tamacc.chuo-u.ac.jp
}

Abstract Information systems of automobiles in the future will have several broadband wireless access systems to the Internet. Among them, DSRC (Dedicated Short Range Communications)[1] is attractive as broadband mobile access system, though coverage will be limited. To extend coverage in effect from viewpoint of users, system can be effective in which terminals share data received from the Internet with other terminals located out of coverage using inter-vehicle communication. In this case, routing protocol of multi-hop inter-vehicle communication network should achieve high throughput with low control overhead when there are many terminals and large amount of data traffic are offered to the network. However, conventional routing protocols using only peer-to-peer communication are difficult to meet these requirements.

This paper presents a cellular-assisted proactive routing protocol for multi-hop inter-vehicle communication network. In the method, each terminal is equipped with both ad hoc mode wireless LAN and cellular system capability. It sends its link state packets to a control node provided in a fixed network using cellular system, and the control node sends link state packets to all terminals using multicast function of cellular system to reduce number of packets transmitted. Simulation using straight road model confirmed that number of transmitted control packets in the proposed method reduces compared with conventional on-demand routing protocol, and amount of reduction increases according to increase of demands for data connections arise in the network.

The original version of this chapter was revised: The copyright line was incorrect. This has been corrected. The Erratum to this chapter is available at DOI: 10.1007/978-0-387-35618-1_37 
Keywords: ITS, In-vehicle terminal, inter-vehicle communication, multi-hop network, cellular system, link state routing protocol

\section{Introduction}

With the emergence of broadband wireless systems including IMT2000, IEEE 802.11 based wireless LAN, DSRC (Dedicated Short Range Communication), capacity of wireless access systems from automobiles to the Internet will increase from conventional tens of kilo bit/sec to mega bit/sec. Among them, hot-spotted network by wireless LAN or DSRC is attractive in that broad bandwidth is available with lower cost than other access systems, although coverage is limited. In order to extend coverage of hot-spotted network in effect from viewpoint of users, a system can be effective in which terminals receive data from the Internet from these systems and they share received data with other terminals located out of coverage using inter-vehicle communication network. As a system for this purpose, authors examine file transfer system using DSRC and multi-hop wireless LAN based inter-vehicle communication network. In the system number of cars participating in multi-hop intervehicle communication network can reach to order of hundred. Transport protocol and routing protocol in the network is required to achieve high throughput with low control overhead. In addition, it should scale to the network with large number of terminals. However, conventional routing protocols of mobile multihop network using only peer-to-peer communication are difficult to meet these requirements.

This paper presents a novel proactive routing protocol called basestation-assisted link state routing protocol, which is suitable for large scale multi-hop inter-vehicle communication network. In this method, each terminal is equipped with wireless LAN and cellular system. Terminals send link state packets to the control node in a fixed network using cellular system, and the control node sends them to all terminals using multicast function of cellular system to reduce number of transmitted control packets.

The rest of the paper is organized as follows. In section 2, the concept of file transfer system using multi-hop inter-vehicle communication network is described, and requirements of routing protocol of the system are presented. In section 3, operation of the proposed method is described. Section 4 presents results of performance evaluation by simulation and finally section 5 concludes the paper. 


\section{Background of research}

\subsection{File transfer systems using inter-vehicle communication}

The concept of the file transfer system using DSRC and multi-hop inter-vehicle communication network examined in this paper is illustrated in Figure 1. From DSRC, cars can receive files containing traffic, map, entertainment information and so on. In this case a car (a) is approaching DSRC, while other car (b) is out of coverage of DSRC. When (a) receives some files via DSRC, it becomes a file sender terminal, and it broadcasts a packet to advertise index of received files to other terminal. If (b) wants advertised files, it issues request to (a) and receives the files from (a) by unicast transport connection. In the rest of this paper, (b) is called file receiver terminal. Since broadcast traffic of file advertisement increases according to increase of file sender terminals, the system has to be designed so that broadcast traffic does not affect performance of file transfer in case there are many file sender terminals.

\subsection{Related works}

There have been many studies of mobile multi-hop network routing protocols $[2,3,4,5,6]$, and they are mainly discussed in MANET(Mobile Ad-hoc NETworks) Working Group of IETF (Internet Engineering Task Force). Among them, ODMRP(On-Demand Multicast Routing Protocol)[4] can work as a routing protocol of the file transfer system described in Section 2.1. In ODMRP, a source terminal with data to send broadcasts JOIN QUERY packets by flooding periodically to other terminals to make multicast group. Terminals that want to join the group send JOIN REPLY packet to the source terminal, and routes between them are constructed. Though ODMRP is simple and efficient protocol, it is in principle suitable to the network with a few source terminals and many multicast receiver terminals. For example, in case that many source terminals can co-exist and each wants to send data to other terminal as described in section 2.1, overhead of ODMRP caused by broadcast of JOIN QUERY packets may not be acceptable.

\section{Base-station-assisted link state routing protocol}

To resolve problems conventional routing protocols described in Section 2.2, this paper presents base-station-assisted link state routing (BALSR protocol. 


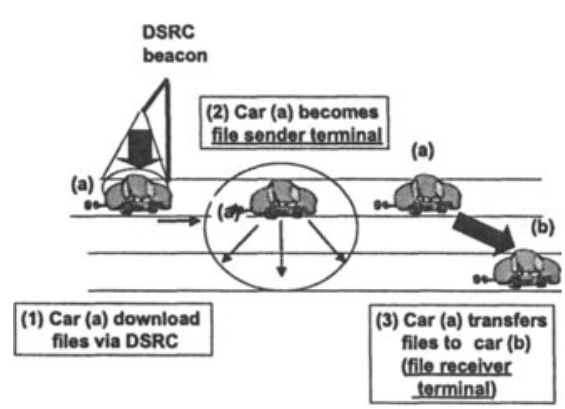

Figure 1. File transfer system using inter vehicle communication

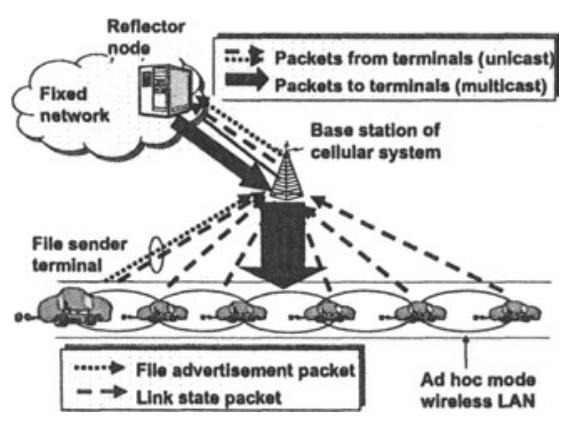

Figure 2. Overview of the proposed system

\subsection{Protocol operation}

It is based on link state routing protocol such as OSPF[7]. Figure 2 shows overview of the system. It is assumed that each mobile terminal is equipped with ad hoc mode wireless LAN and cellular system capability, and both of them support IP (Internet Protocol). Packet types handled in the protocol are listed as follows.

- Hello packet

Packet containing network address of the terminal itself. It is exchanged by wireless LAN.

- Link state packet

Packet containing list of network address of neighbor terminals in wireless LAN. It is exchanged by cellular system.

- File advertisement packet

This packet is generated if a terminal is a file sender terminal(c.f. Section 2.1) and contains indexes of files the terminal has. It is exchanged by cellular system.

\section{- Data packet}

Packet containing user data. It is exchanged by wireless LAN.

Next, configuration of cellular system and fixed network is described. Coverage of a base station in the cellular system is assumed about two kilometers radius, and it supports multicast channel from base station to terminals. Requirements for multicast function in the cellular system are described in detail in Section 3.2. In the fixed network, control nodes called reflector nodes are provided, and they relay control packets 
exchanged between terminals. For terminals within coverage of one base station, one reflector node is assigned. Terminals within coverage area of each base station are informed of network address of the reflector node in advance.

Operation of the protocol is as follows. Each terminal periodically senses neighbor terminals in wireless LAN by exchanging hello packets, and it generates a link state packet and it sends the packet to the reflector node. When a terminal becomes a file sender terminal, a file advertisement packet and a link state packet are sent to the reflector node. When the reflector node receives a file advertisement packet or a link state packet from a terminal, it immediately sends to all terminals using cellular system. In this way, each terminal receives link state packets of all other terminals from the reflector node, and determines routes to other terminals on the multi-hop inter-vehicle network using dijkstra's algorithm. Furthermore, a terminal knows other terminals that received files from DSRC by receiving file advertisement packets.

\subsection{Required functions of cellular system}

The proposed method is designed to send link state packets of multihop network using multicast function of cellular system. To make the proposed method feasible, cellular system is required to support multicast function at data link layer of downlink channel. Among packet switching method in the present cellular systems, GPRS (General Packet Radio Service) supports some of control functions of downlink channel for multicast in PTM (Point-To-Multipoint) service [8]. This can be one of important functions to realize the proposed method. Control function of PTM is presently only specified in the radio interface in [8], and design of upper layer protocols are need to be further investigated.

\section{Performance evaluation}

In this section, performance of the proposed method is evaluated by computer simulation. In the following part, a model of file transfer using inter vehicle communication is defined at first and results of evaluation are shown.

Evaluation model. The model is shown in Figure 3. In this model, road is assumed to have three lanes (a),(b),(c), and DSRC is only available at a spot area of lane (a). Among terminals moving on lane (a), predetermined ratio of terminals receives files via DSRC. Each of terminals that received files become file sender terminal, and it selects the furthest terminal from itself in multi-hop network as a file receiver termi- 
nal. Connection duration time for which one file sender terminal sends packets to one file receiver terminal is assumed to be 20 second. During connection duration time, file sender terminal sends data packet with rate of 50 packet/sec. When connection duration time ended, the file sender terminal selects another file receiver terminal and begins to sending packets

In each lane, a terminal enters from the left hand side and moves to the right hand side at constant speed. Velocity of each terminal is determined when it enters the lane, and it varies between $11.5 \mathrm{~m} / \mathrm{s}$ (about $40 \mathrm{~km} / \mathrm{h}$ ) and $16 \mathrm{~m} / \mathrm{s}$ (about $60 \mathrm{~km} / \mathrm{h}$ ). Average time interval at which a terminal enters a lane is 3.7 second, and thus average distance between newly entered terminal and the precedent terminal is $50 \mathrm{~m}$.

Parameters of ad hoc mode wireless LAN and cellular system are shown in Table 1.

Routing protocol to be evaluated. Based on the evaluation model, two protocols of BALSR and ODMRP are evaluated. Parameters of each protocol are as follows. In the evaluation of BALSR, time interval of hello packet and link state packet is $2.5 \mathrm{sec}$ and $7.5 \mathrm{sec}$ respectively. Link state packet is also sent every $2.5 \mathrm{sec}$ in case that topology is changed within this time interval.

In the evaluation of ODMRP, each file sender terminal sends JOIN QUERY packet by broadcast, and every terminal receiving JOIN QUERY packet sends JOIN REPLY packet to the file sender terminal. Among these terminals the file sender terminal selects the furthest terminal as a file receiver terminal and begins to send data packets. When connection duration time ends, a file sender terminal broadcasts JOIN QUERY packet again and selects another file receiver terminal in the same way. Though ODMRP is mainly designed for multicast communication, performance enhancement for unicast communication is also studied[9], and operation of ODMRP in the evaluation follows description in [9].

Results. Figure 4 shows ratio of data packets successfully delivered to file receiver terminals. Ratio of file sender terminals means number of file sender terminals over number of all terminals on the road. BALSR and ODMRP can achieve data packet delivery ratio of almost $100 \%$ regardless of number of file sender terminal. Next, figure 5 shows number of control packets transmitted by all terminals and a reflector node to construct and maintain routes in BALSR and ODMRP. In results of BALSR, control packets include hello packets and link state packets. In results of ODMRP, control packets include only JOIN QUERY packets. As shown in the figure, BALSR improves control overhead compared 
with ODMRP in case that ratio of file sender terminals is more than $15 \%$ of all terminals. Amount of reduction of control packet increases according to increase of file sender terminals, and it is $50 \%$ at maximum.

These results show that the proposed method achieves the same data packet delivery performance as ODMRP, and reduces control overhead. This feature is effective in case that larger amount of data traffic is offered to the network.

\section{Conclusion}

In this paper, routing protocol for wireless LAN based inter-vehicle communication network is proposed where cellular system is used to exchange control packets. Results of performance evaluation in the straight road model show that the proposed method reduces number of transmitted control packets compared with conventional ODMRP when ratio of terminals with data to send is more than $15 \%$ among all terminals. It is also shown that amount of reduction of control packets reaches $50 \%$ at maximum. This feature will be effective in the environment of inter-vehicle communication in the future to handle large number of data connections. For future work, operation parameters of reflector node will be further evaluated by simulation.

\section{Acknowledgments}

The authors wish to thank Prof. Shigehiko Naoe (Chuo University) and Mr. Tadao Mitsuda (TOYOTA InfoTechnology Center Co., Ltd) for their valuable comments.

\section{References}

[1] ARIB Standard STD-T75 Ver 1.0,"Dedicated short-range communication system" Sep 2001.

[2] Charles Perkins et al., "Ad hoc On-demand Distance Vector (AODV) Routing," Internet-Draft <draft-ietf-manet-aodv-10.txt> Jan 2002.

[3] David B. Johnson et al., "The Dynamic Source Routing Protocol for Mobile Ad Hoc Networks (DSR)", Internet-Draft <draft-ietf-manet-dsr-07.txt> Feb 2002.

[4] Sung-Ju Lee et al., "On-Demand Multicast Routing Protocol", Proc. of IEEE WCNC '99. pp.1298-1302, Sep 1999.

[5] Mario Gerla et al., "Fisheye State Routing Protocol (FSR) for Ad Hoc Networks," Internet-Draft <draft-ietf-manet-fsr-03.txt> Jun 2002.

[6] Amir Qaxyum et al., "Multipoint relaying: An efficient technique for flooding in mobile wireless networks," INRIA Technical Report RR $n^{\circ} 3898$, Mar 2000.

[7] J. Moy, "OSPF Version 2", RFC 2328 Apr 1998. 
[8] ETSI TS 101 350: "Digital cellular telecommunication system (Phase 2+); General Packet Radio Service (GPRS); Overall description of the GPRS radio interface; Stage 2", Feb 2002.

[9] Sang Ho Bae et al., "Unicast Performance Analysis of the ODMRP in a Mobile Ad hoc Network Testbed.," Proc. of IEEE ICCCN 2000. pp.148-153, Oct 2000.

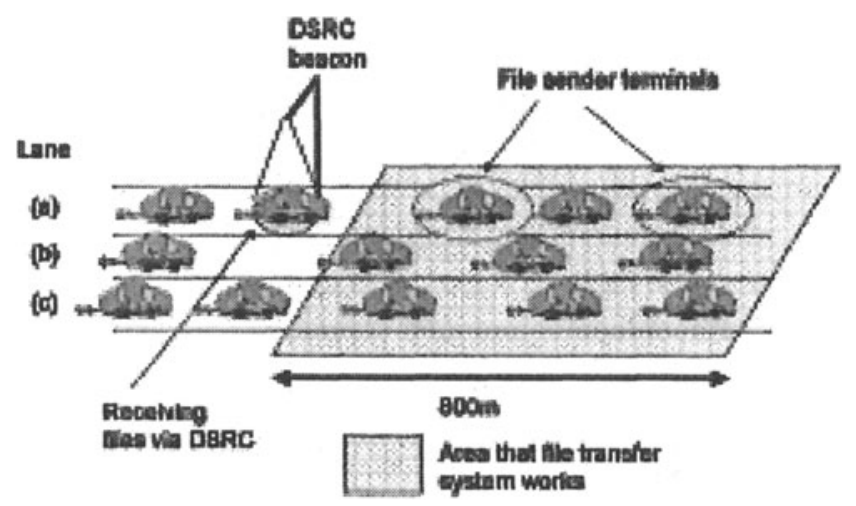

Figure 3. Evaluation model of the file transfer system

Table 1. Parameters of wireless communication systems

\begin{tabular}{ccc}
\hline Type of communication system & Transmission speed & Radius of radio range \\
\hline Ad hoc mode wireless LAN & $11 \mathrm{Mbps}$ & $150 \mathrm{~m}$ \\
Cellular system & $100 \mathrm{kbps}$ & $2000 \mathrm{~m}$ \\
\hline
\end{tabular}

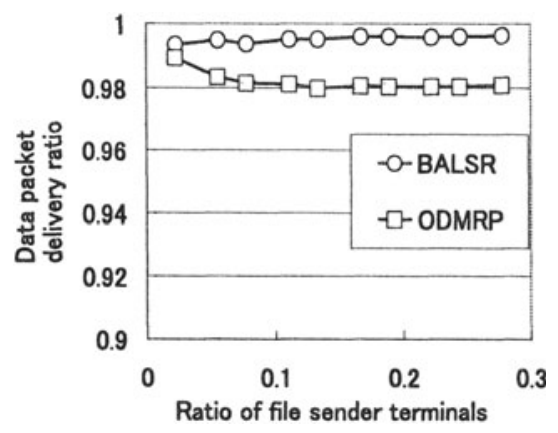

Figure 4. Performance of data packet delivery ratio

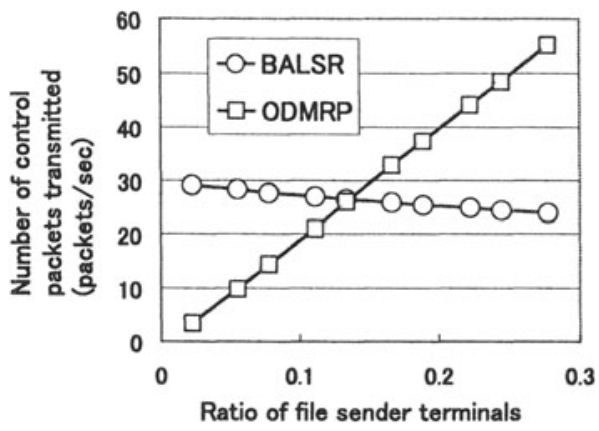

Figure 5. Characteristics of number of transmitted control packets 